\title{
STOCHASTIC OPERATORS AND EXTREME POINTS
}

\author{
Don HAdwin, ERIC Nordgren AND Heydar RADJAVi
}

Abstract. The operators on $L^{p}, 1 \leqslant p<\infty$ that preserve positivity and the constants are shown to have the composition operators as extreme points. In the case of the unit interval with Lebesgue measure they constitute the closed convex hull of these extreme points, but this is not true of all measure spaces.

Mathematics subject classification (2000): Primary 47L07; Secondary 47B33.

Keywords and phrases: positivity preserving operators, extreme points.

\section{REFERENCES}

[1] W. ARveson, Operator algebras and invariant subspaces, Ann. Math., (2) 100 (1974), 433-532. MR0365167 (51 \#1420)

[2] A. IwANiK, Pointwise induced operators on $L_{p}$-spaces, Proc. Amer Math Soc., 58 (1976), 173-178. MR0412883 (54 \#1004)

[3] R.R. PhelPS, Extreme positive operators and homomorphisms, Trans. Amer. Math. Soc., 108 (1963), 265-274. MR0156224 f27 \#6153.

[4] R. SiKORSKI, On the inducing of homomorphisms by mappings, Fund. Math., 36, (1949), 7-22. MR0031535 (11,166a)

[5] R. SiKors KI, Boolean algebras, Second edition, Ergebnisse der Mathematik und ihrer Grenzgebiete, Band 25, Springer-Verlag New York Inc., New York, 1969. MR0242724 (39 \#4053)

[6] A. Sourour, Pseudo-Integral Operators, Trans. Amer. Math. Soc., 253 (1979), 339-363. MR0536952 (80k:47056) 